\title{
Ocean feature models - their use and effectiveness in ocean acoustic forecasting
}

\author{
J. Small, L. Shackleford, G. Pavey \\ Defence Research Agency, Building A22, Winfrith Technology Centre, Winfrith Newburgh, Dorchester, Dorset, DT2 8XJ, UK
}

Received: 20 December 1995/Revised: 17 June 1996/Accepted: 30 July 1996

\begin{abstract}
The aim of this paper is to test the effectiveness of feature models in ocean acoustic forecasting. Feature models are simple mathematical representations of the horizontal and vertical structures of ocean features (such as fronts and eddies), and have been used primarily for assimilating new observations into forecasts and for compressing data. In this paper we describe the results of experiments in which the models have been tested in acoustic terms in eddy and frontal environments in the Iceland Faeroes region. Propagation-loss values were obtained with a 2D parabolic-equation (PE) model, for the observed fields, and compared to PE results from the corresponding feature models and horizontally uniform (range-independent) fields. The feature models were found to represent the smoothed observed propagation-loss field to within an rms error of $5 \mathrm{~dB}$ for the eddy and $7 \mathrm{~dB}$ for the front, compared to $10-15-\mathrm{dB}$ rms errors obtained with the range-independent field. Some of the errors in the feature-model propagation loss were found to be due to high-amplitude 'oceanographic noise' in the field. The main conclusion is that the feature models represent the main acoustic properties of the ocean but do not show the significant effects of small-scale internal waves and finestructure. It is recommended that feature models be used in conjunction with stochastic models of the internal waves, to represent the complete environmental variability.
\end{abstract}

\section{Introduction}

In the ocean, spatial variability occurs on a wide range of scales from basin-scale gyres to internal waves with wavelengths of $100 \mathrm{~m}$, and further down to molecular scale. Theory and observations have shown that the energy of the ocean variability decreases as the scales of

Correspondence to: J. Small motion decrease (Pedlosky, 1987), with maximum energy at the mesoscale (from $\sim 10-\sim 100 \mathrm{~km}$, dependent on the location). Mesoscale features include fronts and eddies. The aim of this paper is twofold: to investigate the relative acoustic impact of ocean scales ranging from the mesoscale to the internal-wave scale, using high-resolution observed temperature data; and to test the effectiveness of simple feature models in representing the real data.

Feature models are simple mathematical representations of the mesoscale structure in ocean features (such as fronts and eddies), and have been developed for use in forecasting ocean models such as the Harvard Gulf Stream model (Spall and Robinson, 1990), and the Forecast Ocean Atmosphere Model, FOAM (Heathershaw and Foreman, 1994). Feature models are useful as a means of compressing gridded data for transmission of data to ships at sea, and for assimilating data into ocean models (Smeed, 1995). The compression is achieved by decomposing a large set of gridded data into a smaller set of feature parameters, such as radius and centre position of eddies, and amplitudes of climatological empirical orthogonal functions (EOFs) (Smeed and Alderson, 1995). These parameters can then be input into a simple feature model at sea to reconstruct the data. For assimilation purposes, feature models are useful in linking the readily available remotely sensed surface data [such as sea-surface temperature (SST) by satellite radiometer] to less common insitu data from ships, to model the subsurface structure in a manner that maintains the characteristic structures of ocean features (Smeed, 1995).

For ocean-acoustic forecasting purposes, the question arises of whether the feature models are adequate representations of gridded data in terms of their effect on sound propagation. This will depend on, a, whether the feature models adequately represent the mesoscale spatial variations, and $b$, whether the small-scale activity which is present in the ocean but not represented in feature models has a significant effect on acoustic propagation over mesoscale distances.

Previous studies using a combination of deterministic and stochastic ocean models have implied that small-scale 
random internal-wave activity has a medium effect on acoustic propagation [where a medium effect is defined as a 5-10-dB alteration in propagation loss (Small, 1994, Chin-Bing et al., 1994)]. This paper will seek to confirm this with observed temperature data. The data used here is a combination of high-resolution thermistor chain data and lower-resolution deep XBT (expendable bathythermograph) data, obtained in the Iceland-Faeroes Front (IFF) region.

To enable complete high-resolution cross-sections of sound-speed data to be made down to the sea-bed, a novel method has been developed to extrapolate the chain data below the depth of the chain, using knowledge of the XBT data at those depths. The data created using this method is then used for acoustic computations in comparison with feature models of the data, and range independent (i.e. where the environmental conditions are horizontally uniform) scenarios.

\section{Description of the data analysis and modelling methods}

\subsection{Introduction to the physical oceanography}

This paper is concerned with data from the IFF region. Useful reviews of the oceanography of the IFF region have been given in Hopkins (1991) and Scott and Lane (1991). In summary, the region is characterised by a front which is topographically linked to the Iceland-Faeroes ridge. The topography of the region is shown in Fig. 1 [from the Earth Topography and Ocean Bathymetry Database (ETOP05), issued by the National Oceanic and Atmospheric Administration, 1993]. The front separates the Atlantic waters to the south from the Norwegian Sea waters to the north, with the ridge preventing overflow of deep Norwegian Sea water into the Atlantic. A common feature of the region is a bulge of warm near-surface water which extends from $-9^{\circ}$ to $-11^{\circ} \mathrm{E}$ and from $64^{\circ} 30^{\prime} \mathrm{N}$ to $66^{\circ} \mathrm{N}$. This feature can be seen on an advanced very high resolution radiometer (AVHRR) SST image of the area shown in Fig. 2, taken on 14 May 1988, by the NOAA (National Oceanic and Atmospheric Administration) 9 satellite. The formation of the warm intrusion has been hypothesised to be achieved by the penetration of narrow jets of warm water northwards, often in conjunction with the southward intrusion of cold jets (Scott and McDowell, 1990: on Fig. 2 this occurs at about $64^{\circ} 40^{\prime} \mathrm{N}, 10^{\circ} 30^{\prime} \mathrm{W}$ ). The cold jets often separate to become isolated cold eddies. The data to be presented illustrate one of these cold eddies, and a section of the front towards its western end.

\subsection{The oceanographic data}

For these experiments observed temperature data from surveys of the IFF region undertaken by the Defence Research Agency (DRA) were used. High-resolution temperature slices were available from the DRA thermistor chain (Lane and Scott, 1986), which samples at $50 \mathrm{~Hz}$ as it is towed through the water at around $2 \mathrm{~m} \mathrm{~s}^{-1}$, with 100 temperature pods and 10 pressure pods arranged along the 400-m quasi-vertical chain. The data were subsampled to one profile every $45 \mathrm{~s}$, for ease of handling. In addition to the chain records, XBT data was used to give an idea of the deeper temperature structure, and data from 4 CTD (conductivity temperature depth) sensors positioned on the chain gave information on the salinity structure. The XBTs were cast between one and two times an hour. The DRA thermistor chain data was used for this study as it provided an opportunity for the small-scale internal activity

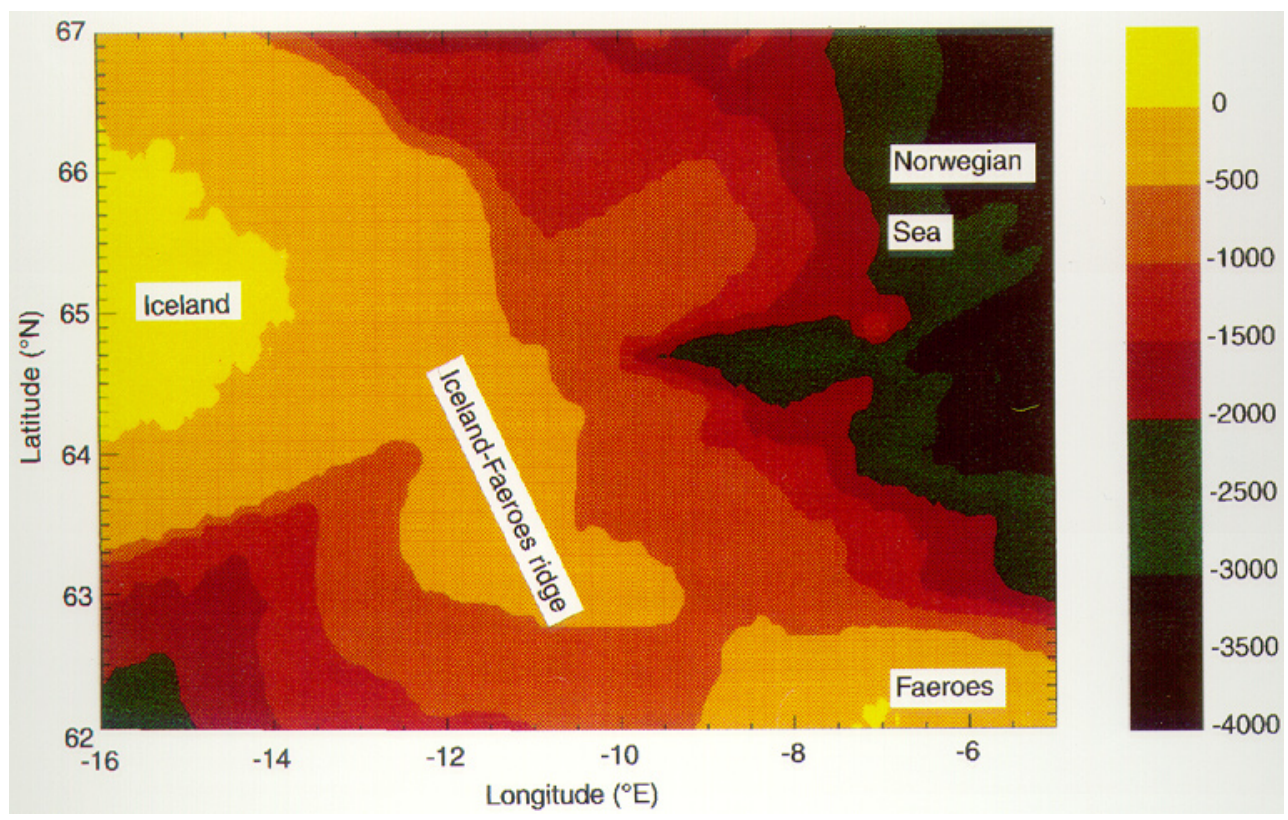

Fig. 1. Bathymetry of the IcelandFaeroes region, indicating the Iceland-Faeroes ridge. From ETOP05. The depth scale at 500-m intervals is indicated in the colourbar 


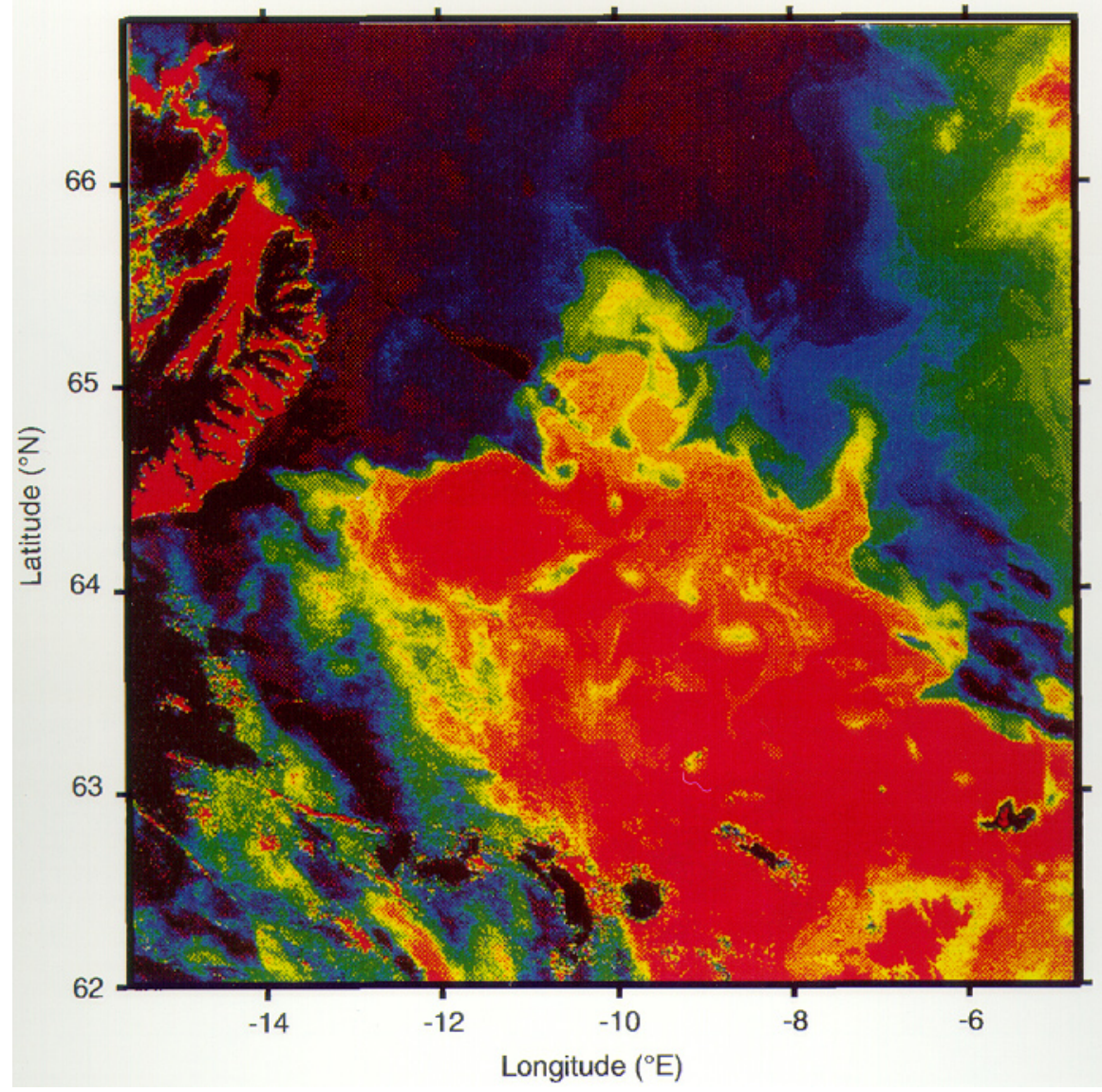

Fig. 2. Satellite AVHRR of the IcelandFaeroes Frontal zone, indicating the front, the warm intrusion and a cold jet occurring at $-11^{\circ} \mathrm{E}, 64^{\circ} 30^{\prime} \mathrm{N}$. Taken on 14 May 1988. Here warm tempera tures are red, cold temperatures blue. The bottom left corner of the image is obscured by cloud to be studied. The chain subsample resolution of $45 \mathrm{~s}$ corresponds to one profile every $90 \mathrm{~m}$, and thus allows most of the internal-wave scales to be resolved (the minimum resolvable wavelength being $180 \mathrm{~m}$ ).

Figure 3 shows the two temperature structures measured by thermistor chain and used in this paper. Fig. 3a is a section through a cold winter eddy cut off from the Iceland-Faeroes front, giving rise to temperature differences around $5^{\circ} \mathrm{C}$ around $100-\mathrm{m}$ depth, surveyed in May 1988 (Scott, 1991). Figure 3b shows the front at its western end surveyed in July 1991 (Geddes and Scott, 1993), showing temperature changes of up to $6^{\circ} \mathrm{C}$ over $5-10 \mathrm{~km}$ at $200-\mathrm{m}$ depth. The data is plotted as a function of actual depth (derived from knowledge of the depth along the thermistor chain and tow speed, and information from pressure sensors along the chain) and tow range.

\subsection{The feature models}

Feature models describe oceanographic features mathematically using a number of variable parameters, indicating size, shape and position of the feature, which are derived from available observations or climatology. The feature models used here are now outlined.

\subsubsection{The cold eddy}

Here only one horizontal dimension is modelled (the along tow direction), which is taken to be the $x$ direction. The temperature in the feature model $T_{f}(x, z)$ at any point within the eddy is given by (Smeed and Alderson, 1995):

$T_{f}(x, z)=T_{1}(z)+\left(T_{2}(z)-T_{1}(z)\right) \exp \left(-\frac{r^{2}}{L(z)^{2}}\right)$,

where

$r^{2}=\left(x-x_{0}\right)^{2}$,

and the parameters are the location of the centre $\left(x_{0}\right)$, the e-folding radius $L$, the temperature profile of the surrounding water $T_{1}(z)$ and the profile at the eddy centre $T_{2}(z)$. This formula assumes a Gaussian decay of the eddy amplitude with horizontal distance from the centre. The parameters are illustrated schematically in Fig. 4a for a cold eddy. In this study the radius $L$ is allowed to vary linearly with depth, introducing another parameter.

In these experiments, the parameters are found using simple search techniques [more sophisticated techniques have been discussed in Smeed and Alderson (1995): for this study of 2D fields, however, simple search techniques were found to be sufficient]. First the mean vertical profile of the background outside the eddy $T_{1}(z)$ is obtained by 


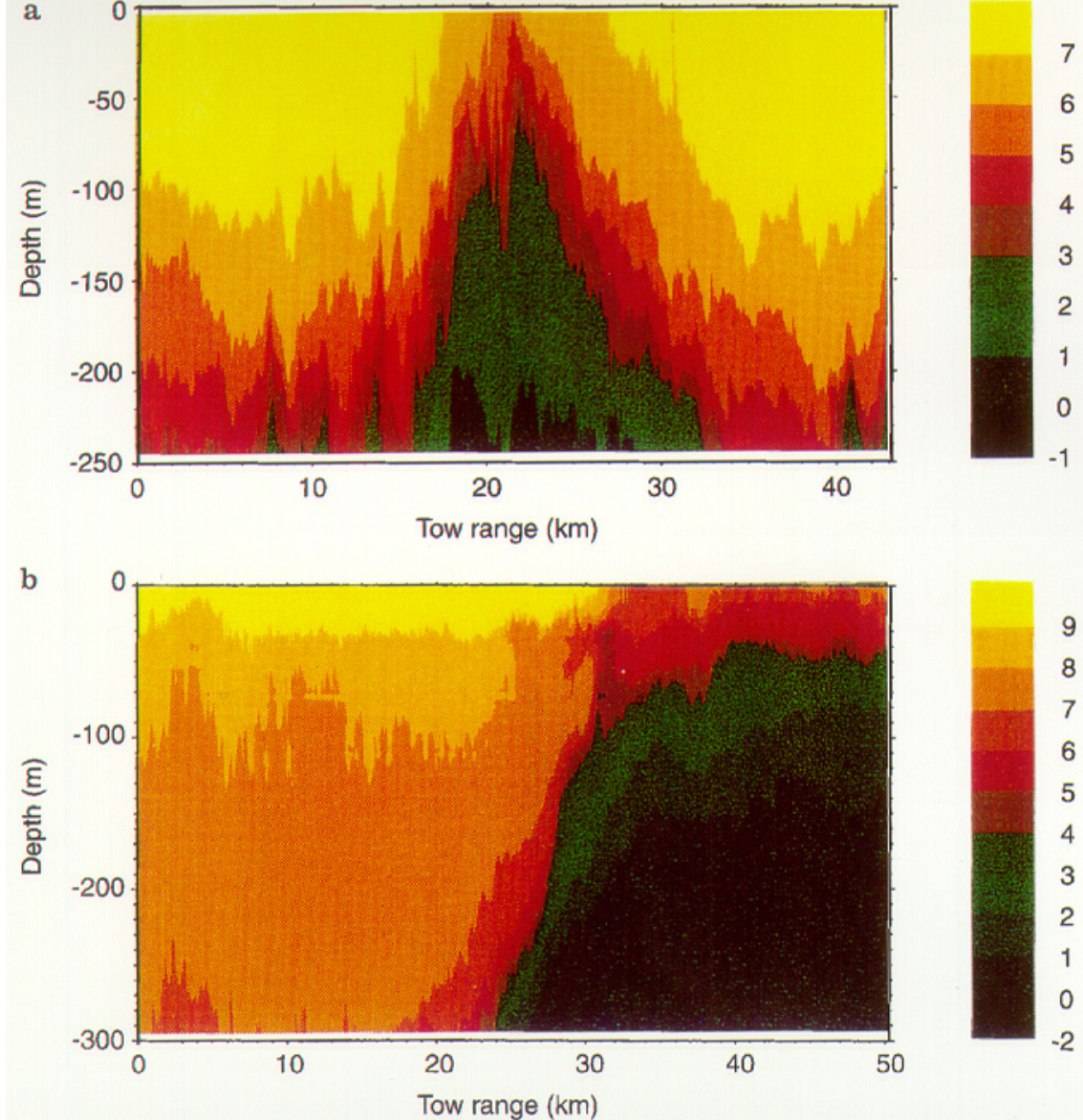

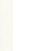

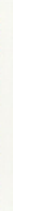

Fig. 3a, b. Thermistor chain temperature sections through two oceanographic features: a a cold eddy in winter; b the western end of the Iceland-Faeroes front in summer. The temperature scale in ${ }^{\circ} \mathrm{C}$ is shown on the colour-bars horizontally averaging the thermistor chain and XBT data outside the eddy. Then the thermistor-chain data is horizontally smoothed over a moving window of 10 data points $(900 \mathrm{~m})$ to provide a smooth field $T(x, z)$ of the eddy for analysis of the parameters (this is done to eliminate the small-scale activity which would corrupt the parameter estimation). Next the position of the centre of the eddy is found by searching for the position where the magnitude of the temperature difference $\left(T(x, z)-T_{1}(z)\right)$ is greatest. This is done at the depth of each thermistor along the chain, and the values averaged to give a mean $x_{0}$.

The radius is determined by searching for the positions where the temperature difference satisfies the following equation:

$T(x, z)-T_{1}(z)=\frac{1}{e}\left(T\left(x_{0}, z\right)-T_{1}(z)\right)$

(this is the definition of the e-folding radius, and is derived from Eq. 1; the search is made either side of the eddy centre and the mean taken). This is done at each depth, and $L(z)$ is set to $\left(x-x_{0}\right)$. A straight line is fitted to the function $L(z)$ of the form

$L(z)=A+B z$

where $A$ and $B$ are the determined coefficients; thus the radius is described by these two coefficients.
The temperature profile within the eddy $T_{2}(z)$ is taken to be just the temperature profile $T\left(x_{0}, z\right)$ for the depths down to the bottom of the chain $(z=B)$. Below the chain, the nearest XBT to the eddy centre is used to calculate the profile. If $T_{x}(z)$ is the XBT profile, and $r$ is the horizontal distance of the XBT cast from the eddy centre, then the profile $T_{2}(z)$ for points below the chain will be given by:

$T_{2}(z)-T_{1}(z)=\frac{T_{x}(z)-T_{2}(z)}{\exp \left(-r^{2} / L^{2}\right)}, \quad z>B$,

which is deduced from Eq. 1.

Using these parameters a feature model is created of the eddy. Fig. 5a shows the feature model of the cold eddy illustrated in Fig. 3a. The feature model was constructed at a resolution of $5 \mathrm{~km}$, simulating a likely resolution for future forecast models of the region [the internal Rossby radius, a characteristic mesoscale lengthscale, is around $16 \mathrm{~km}$ in the Iceland-Faeroes (Maskell et al., 1992)].

For this eddy, salinity data was not available, and the salinity has been modelled by assuming a linear relationship between temperature $T$ and salinity $S$, taken from Scott and McDowell (1990):

$S=a T+b$, 
a

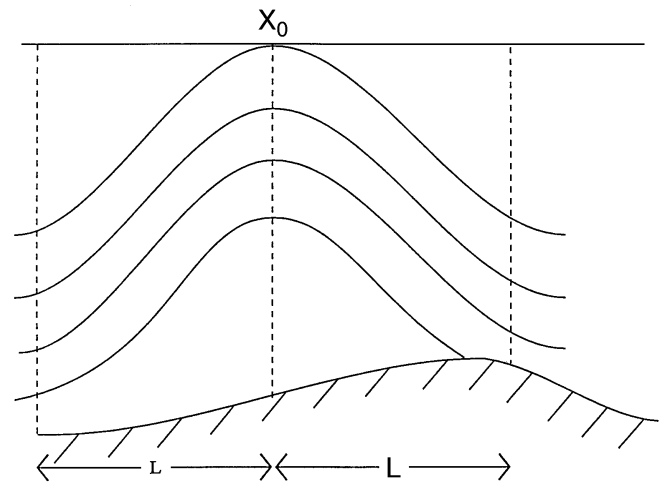

b

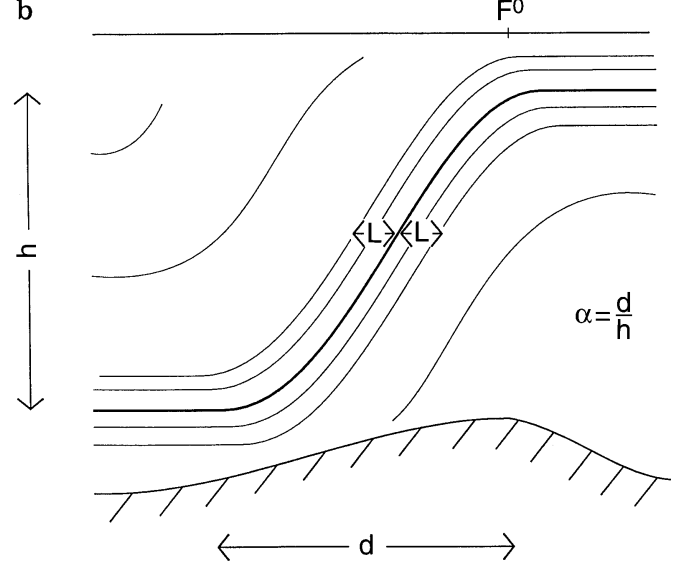

Fig. 4a, b. Schematic illustrations of feature parameters: a cold eddy, showing the centre $x_{0}$ and radius $L$, and $\mathbf{b}$ front, showing the surface position $\mathrm{F}^{0}$, width $L$, and slope $\alpha$

where $a=0.05 \mathrm{ppt}^{\circ} \mathrm{C}^{-1}$ and $b=34.8 \mathrm{ppt}$. This relationship was also used for the interpolation of the data to be described later.

\subsubsection{The western end of the Iceland-Faeroes front}

A front is basically a transition region between two water masses. It is characterised by its slope in the vertical plane, its width, and its surface position. A feature model of a front using these characteristics as parameters is described here, again using $x$ as the along tow (and across front) direction.

The temperature $T_{f}(x, z)$ at any point in the feature model can be defined as (Smeed and Alderson, 1995):

$T_{f}(x, z)=T_{1}(z)+f(s(x, z), l)\left(T_{2}(z)-T_{1}(z)\right)$,

where $T_{1}(z)$ and $T_{2}(z)$ are the profiles of the two water masses, $s(x, z)$ is the horizontal minimum distance of the point $(x, z)$ from the front (taking a particular isotherm surface to represent the front), $l$ is the frontal width and $f(s, l)$ is given by

$f(s(x, z), l)=\frac{(1+\tanh (s / l))}{2}$.

The function $f(s, l)$ is chosen to vary from 0 to 1 as $s$ goes from $-\infty$ to $+\infty$, with the change occurring mainly over the width $l$. Hence, from Eq. 7, $T_{f}(x, z)$ will vary from $T_{1}(z)$ to $T_{2}(z)$ as $s$ goes from $-\infty$ to $+\infty$. The function $s$ can be parameterised in terms of the slope of the front, and the position of the front at the surface, as will be described.

Once again the parameters are obtained using simple searching and averaging techniques. The water-mass profiles are obtained by horizontally averaging the thermistor-chain and XBT data on either side of the front away from the front. The slope and surface position of the front were obtained from visual inspection of a contour of the chain data. Knowledge of the slope and surface position of the front leads to a linear approximation to the horizontal position of the front at depth, $F(z)$ :

$F(x)=F^{0}+\alpha z$,

where $F^{0}$ is the surface position and $\alpha$ the slope (illustrated in Fig. $4 b$, which shows the parameters on a schematic front). The distance $s(x, z)$ is then just given by

$s(x, z)=x-F(z)$.

The width of the front was obtained by searching for the positions $x_{n}$ at a particular depth level where

$T\left(x_{n}, z\right)-T_{1}(z)=\frac{1}{2}(1+\tanh (1))\left(T_{2}(z)-T_{1}(Z)\right)$

(deduced from Eqs. 7 and 8: the search is made either side of the front and the mean taken). The width at that depth is then given as $\left(x_{n}-F(z)\right)$. This is done at each vertical level and the values at each depth point are averaged to give a mean width $l$. Using this method, a feature model of the front illustrated in Fig. $3 \mathrm{~b}$ was made, as shown in Fig. 5b.

For this feature, the salinity was modelled using information from a $\mathrm{T}-\mathrm{S}$ diagram of the data obtained from the CTD and thermistor chain values (Geddes and Scott, 1993) (Fig. 6). For the cold-water mass, a linear fit of the form of Eq. 6 was made to the data, with $a=$ $-0.033 \mathrm{ppt}^{\circ} \mathrm{C}^{-1}$ and $b=34.82 \mathrm{ppt}$, while for the warm water, a constant value of $S=35.15 \mathrm{ppt}$ was assumed. These linear fits are shown in Fig. 6, along with the approximation used for the cold eddy.

\subsection{Extrapolation and interpolation of the data using a correlation technique}

In order to assess whether the small-scale activity in the ocean diminishes the acoustic effectiveness of feature models, cross-sections of the observed data at high resolution (900 m horizontally, $4 \mathrm{~m}$ vertically) have been used. Unfortunately the high-resolution chain data extend only about 250-300 m deep, and below this only coarse-resolution XBT data was available. To remedy this problem, the small-scale activity below the chain data has been simulated using a simple extrapolation/interpolation technique.

The extrapolation method involves firstly the calculation of the amplitude of the small-scale activity in the thermistor-chain data. Here the feature model of the data (calculated as above) is subtracted from the chain data, to give the perturbation field $T_{p}(z)$ of submesoscale activity. 

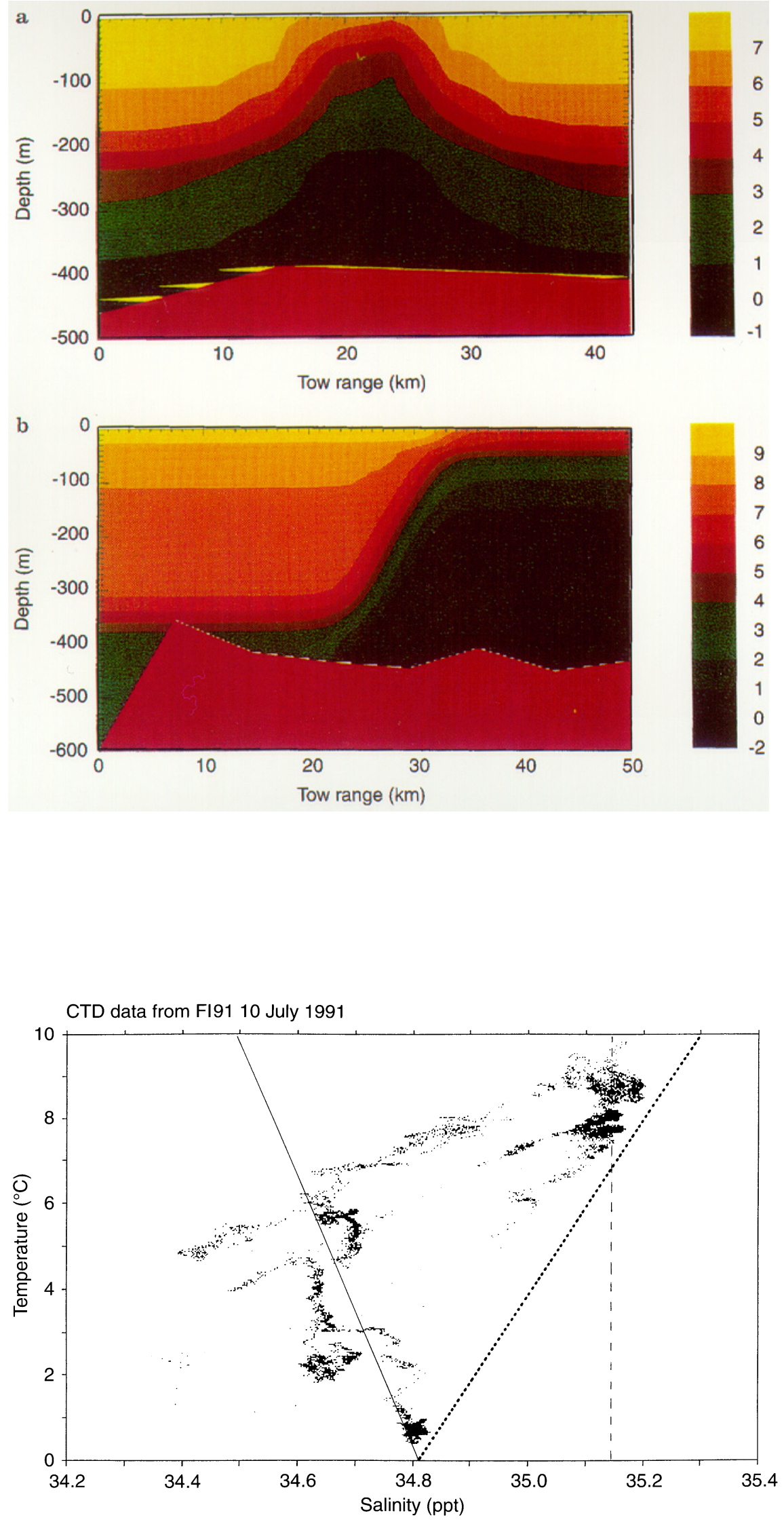

Fig. 5a, b. Feature models of the features shown in Fig. 3: a the cold eddy; $\mathbf{b}$ the western end of the Iceland-Faeroes front. The temperature scale in ${ }^{\circ} \mathrm{C}$ is shown on the colour-bars
Fig. 6. Temperature-salinity (TS) diagram for the frontal environment, obtained from four CTD sensors on the thermistor chain. Indicated on the diagram are the approximations to the TS relationship used for the frontal models (solid line for cold side and dashed line for the warm side), and also the approximation used for the eddy models (dotted line) 
Then, for each profile, the perturbation at the bottom of the chain $T_{p}(B)$ is identified. The perturbation $T_{p}(z)$ below the chain is then modelled as

$T_{p}(z)=T_{p}(B) \exp \left(-\frac{(z-B)^{2}}{C^{2}}\right), \quad z>B$,

where $C$ is a correlation lengthscale. Effectively this method decays (or decorrelates) the amplitude of the internalwave activity to a negligible amount over the distance $C$.

The choice of correlation scale has been based partly on dynamical theory. Calculation of the linear internalwave modes for the background fields investigated in this experiment [using a numerical shooting technique, NAG D02KEF (Numerical Algorithm Group, 1993) to solve the Taylor-Goldstein equation with no shear (Garrett and Munk, 1972)] showed that the amplitude of the modes decreased to zero below $250 \mathrm{~m}$ over scales of around $100 \mathrm{~m}$, with no extra nodes below $250 \mathrm{~m}$. Hence a value of $100 \mathrm{~m}$ has been chosen for the decorrelation scale.

The final field is then formed of the chain data down to the chain bottom, and below this the sum of the feature model data and the profiles $T_{p}(z)$. The temperature fields created using this method, of the eddy and the front, are illustrated in Fig. 7. Salinity values for the eddy were simulated using the linear T-S relationship of Sect. 2.3, and for the front the salinity field was approximated by

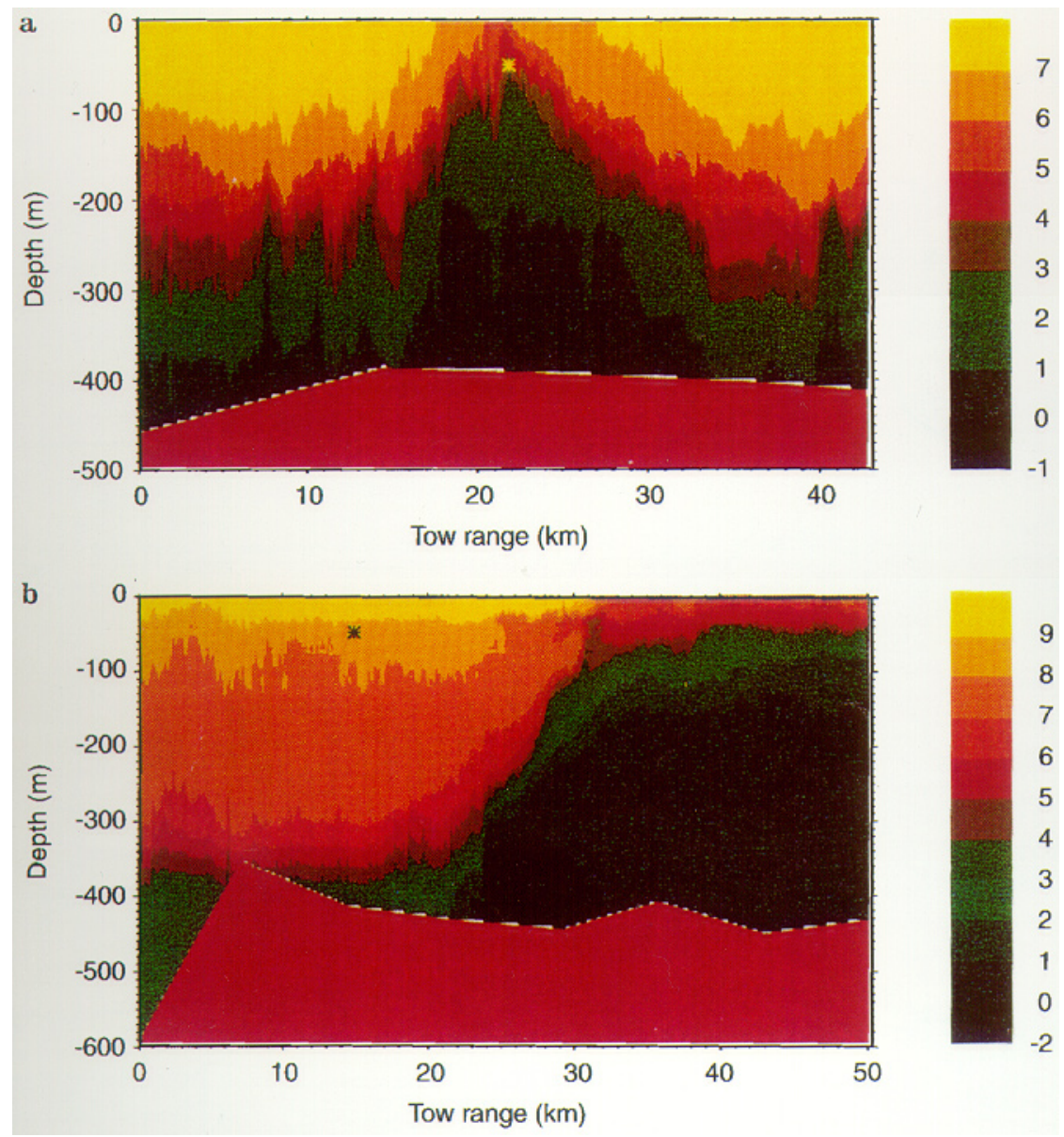

that from the feature model. Therefore, for the front, the difference between the feature model and the real data is in the temperature field alone. The top $250-300 \mathrm{~m}$ of the plots in Fig. 7 are identical to those in Fig. 3. Comparison of Figs. 5 and 7 show that the feature model does represent the large-scale structure of the features, whilst not including any small internal-wave features.

\section{The acoustic experiments}

\subsection{Design of the acoustic modelling experiments}

In order to test the acoustic effectiveness of feature models, modelling was done of the sound propagation through three fields for each feature: the high-resolution observed field, the feature model and a range-independent (horizontally uniform) field that does not include the feature. Comparison of the results should indicate, a, to what extent a feature model is an improvement over the range-independent case in representing the real data, and $b$, what effect small submesoscale waves have on the acoustics.

The acoustic effects were assessed in terms of the variation in propagation loss (PL), defined in terms of the sound intensity $I$ by

$$
\mathrm{PL}=10 \log _{10} \frac{I_{0}}{I}
$$

Fig. 7a, b. Temperature cross-sections down to the seabed for the two environments: $\mathbf{a}$ the cold eddy and $\mathbf{b}$ the front. The temperature scale in ${ }^{\circ} \mathrm{C}$ is shown on the colour-bar. The position of the acoustic source used for the acoustic computations is marked by a yellow asterisk in a and a black asterisk in b 
Table 1. Geoacoustic parameters for the Iceland-Faeroes environment

\begin{tabular}{llllll}
\hline Layer type & $\begin{array}{l}\text { Thickness } \\
(\mathrm{m})\end{array}$ & $\begin{array}{l}\text { Density } \\
\left(\mathrm{kg} \mathrm{m}^{-3}\right)\end{array}$ & $\begin{array}{l}\text { Attenuation } \\
(\mathrm{dB} / \lambda)\end{array}$ & $\begin{array}{l}\text { Sound speed } \\
\left(\mathrm{m} \mathrm{s}^{-1}\right)\end{array}$ & $\begin{array}{l}\text { Sound-speed } \\
\text { gradient } \\
\left(\mathrm{m} \mathrm{s}^{-1} \mathrm{~m}^{-1}\right)\end{array}$ \\
\hline $\begin{array}{l}\text { Sediment } \\
\text { Sub-bottom }\end{array}$ & 160 & 1.85 & 1.45 & $1470-1530$ & 0.37 \\
\hline
\end{tabular}

where $I_{0}$ is a reference intensity $1 \mathrm{~m}$ from the source. Hence a reduction in intensity corresponds to an increase in propagation loss. The propagation loss was computed using the acoustic parabolic equation (Jensen et al., 1994) model PAREQ (Jensen and Martinelli, 1985). PAREQ is a range-dependent model which allows horizontal variability in the environment, treats the surface as a perfect reflector, and models the bottom as a two-layer system of sediment-bottom and subbottom. Geoacoustic parameters for the model were obtained from previous studies of the Iceland-Faeroes ridge region (S Marks, personal communication), and are listed in Table 1.

Here $\lambda$ is the sound wavelength. The seabottom in these experiments lies between 400 and $600 \mathrm{~m}$ deep. Computational range steps $\Delta r$ for PAREQ were $15 \mathrm{~m}$ for the $50-\mathrm{Hz}$ case and $1.8 \mathrm{~m}$ for $400 \mathrm{~Hz}$. These values satisfied the usual criteria for convergence (namely $r<\lambda$ ), and comparisons of the results against those obtained with smaller range steps (not illustrated) by the authors also found the results to be converged. The output resolution of the acoustic model is $150 \mathrm{~m}$ in the horizontal and $4-6 \mathrm{~m}$ in the vertical.

The acoustic impact of the feature models was assessed by analysing propagation loss at 50 and $400 \mathrm{~Hz}$ for a source placed at 50-m depth. For the cold eddy, the source was placed in the middle of the eddy (range $22 \mathrm{~km}$ in Fig. 7a, marked with a yellow asterisk), and for the front, the source was placed about $10 \mathrm{~km}$ to the left of the front (range $15 \mathrm{~km}$ in Fig. $7 \mathrm{~b}$, marked with a black asterisk).

Sound-speed values were calculated from the temperature and salinity using the Chen and Millero (1977) empirical equation. Plots of the sound-speed profiles (SSPs) used for the feature model at the centre of the eddy and outside the eddy are shown in Fig. 8a, together with a profile on the eddy boundary, while Fig. $8 \mathrm{~b}$ shows the feature model SSPs either side of the front, and two SSPs located in the front. From Fig. $8 \mathrm{a}$ it can be seen that the effect of the eddy is significantly to reduce the sound speeds (by up to $20 \mathrm{~m} \mathrm{~s}^{-1}$ ) when compared with the background, and to remove the 100-m surface duct. Across the front (Fig. 8b), sound-speed changes of up to $30 \mathrm{~m} \mathrm{~s}^{-1}$ occur. On the warm side of the front, an increase in sound speed with depth occurs below $50 \mathrm{~m}$, giving rise to a sound channel axis at $50 \mathrm{~m}$, while on the cold side the axis occurs deeper, around $200 \mathrm{~m}$. For the feature model and highresolution cases the seabottom used was as indicated in Figs. 5 and 7, while for the range-independent case the seabottom was assumed to be constant at the water depth of the source location: 400 and $390 \mathrm{~m}$ for the front and eddy, respectively.

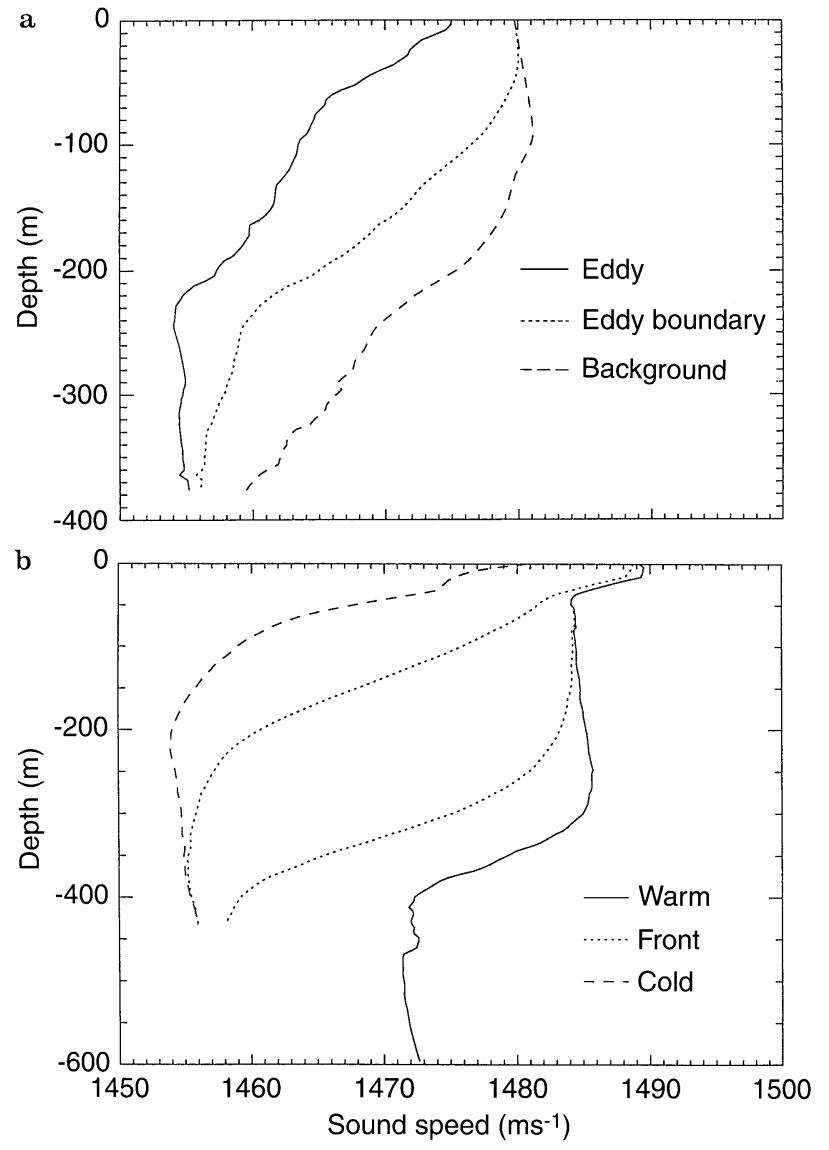

Fig. 8a, b. Sound-speed profiles (SSPs) taken from the feature models: a SSPs at the eddy centre, in the background and on the eddy boundary; b SSPs on the warm and cold side of the front and two SSPs from within the front

\subsection{Acoustic Results}

\subsubsection{Eddy environment}

Some of the results can be seen in Fig. 9a, b, which compares the propagation loss through the SSPs of the real eddy field with those of the feature model and the rangeindependent case, for frequencies 50 and $400 \mathrm{~Hz}$, and for a source and receiver depth of $50 \mathrm{~m}$. At $50 \mathrm{~Hz}$ (Fig. 9a) the general pattern of propagation loss is similar in all three cases, although the feature model follows the observed case most closely up to around $15 \mathrm{~km}$. At $400 \mathrm{~Hz}$ (Fig. 9b) the range-independent case shows higher energy levels (low propagation loss), whereas the feature model correctly predicts more propagation loss as the sound passes 

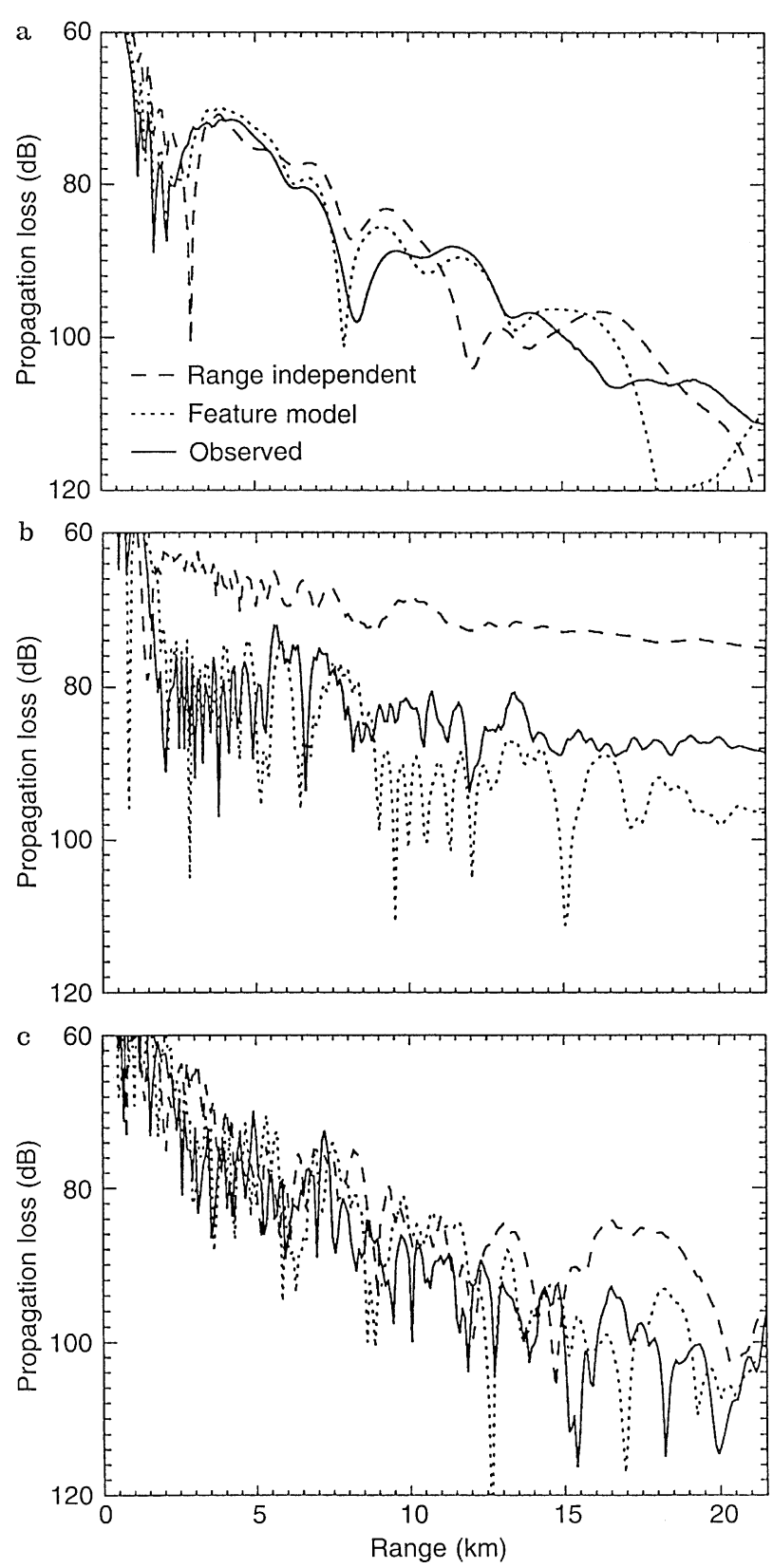

Fig. 9a-c. Propagation-loss values obtained through the eddy environment: a frequency $50 \mathrm{~Hz}$, source and receiver depth $50 \mathrm{~m}$; b frequency $400 \mathrm{~Hz}$, source and receiver depth $50 \mathrm{~m}$; c frequency $400 \mathrm{~Hz}$, source depth $50 \mathrm{~m}$ and receiver depth $150 \mathrm{~m}$

through the cold eddy. However, there are quantitative differences between the observed and the feature-model cases. The feature model, although clearly better than the range-independent background profile, still gives peak errors of up to $20 \mathrm{~dB}$ relative to the observed curve, largely due to the occurrence of nulls of intensity (spikes of high propagation loss) in the curves. These nulls are mainly due to modal interference, and the effects of the internal waves is to alter the position of the nulls, creating large differences between the two fields. [For a finite bandwidth, it has been speculated that these nulls may be smoothed out (Harrison and Harrison, 1995). These authors suggested that one method of simulating the effect of a finite bandwidth is to smooth the output from a single-frequency model using a running mean, with the window size equal to $\alpha$ times the range, where $\alpha$ is the bandwidth as a fraction of the mean frequency. For this study, however, the resolution of the acoustic model output $(150 \mathrm{~m})$ is too high to necessitate smoothing to simulate finite bandwidths of , say, $\mathrm{O}(1 \%)$ of the frequency. However, in the next section smoothing is done just to compare gross-scale features of the acoustic output.]

For a deeper receiver at $150 \mathrm{~m}$, for frequency $400 \mathrm{~Hz}$ (Fig. 9c), the differences between the fields are less notable, although the range-independent case diverges from the other two cases beyond $15 \mathrm{~km}$. An overall view of the propagation is shown in Fig. 10, which illustrates the 2D propagation through the three fields, for a frequency of $400 \mathrm{~Hz}$ and a source at 50-m depth. This shows clearly that the feature model is an improvement over the range independent case, which predicts significant surface channelling not present in the observed case. In the observed and feature-model cases, the sound is refracted downwards as it passes through eddy water which rapidly cools (sound speed decreases) with depth (Fig. 8a), following Snell's law (Urick, 1983).

Table 2a quantifies the above results. Rms differences in propagation loss over the whole field are shown relative to the observed case, for the feature-model and the rangeindependent fields. In addition, the percentage of the field in error (relative to the observed case) by over $10 \mathrm{~dB}$ is shown. This is done for frequencies of 50 and $400 \mathrm{~Hz}$, with the source at $50 \mathrm{~m}$.

The unsmoothed results indicate the improved skill of the feature model over the range-independent case in representing the observed data, particularly at the higher frequency, with lower values of rms error and of percentage of the field over $10 \mathrm{~dB}$ different. However, a straight rms difference gives little indication of the nature of the differences. In order to show whether the differences are from spikes (sharp nulls of intensity) or consistent levels of difference, the propagation-loss data was smoothed to remove the spikes, using a moving average of 10 horizontal points $(1.5 \mathrm{~km})$, and the rms calculations redone. The results from this are shown on the right-hand side of Table 2a. Generally the smoothing has more effect on the feature-model results, improving the skill, and thus showing that many of the differences between the feature-model and the observed propagation-loss fields are due to random noise. In particular, the percentage of the featuremodel field over $10 \mathrm{~dB}$ in error is reduced by smoothing to about a quarter of its unsmoothed value at $400 \mathrm{~Hz}$. In contrast, smoothing has little effect on the skill of the range-independent case (especially at $400 \mathrm{~Hz}$ ), implying that the errors are more consistent. This confirms the visual analysis discussed above. For the smoothed results, the feature model represents the observed propagation loss values to within $5 \mathrm{~dB}$ at 50 and $400 \mathrm{~Hz}$, compared to 7- and $10-\mathrm{dB}$ errors at 50 and $400 \mathrm{~Hz}$, respectively, for the range-independent case. The smoothing has less effect overall at the lower frequency, as the original fields are already smoother at this frequency (compare Fig. 9a and b). 

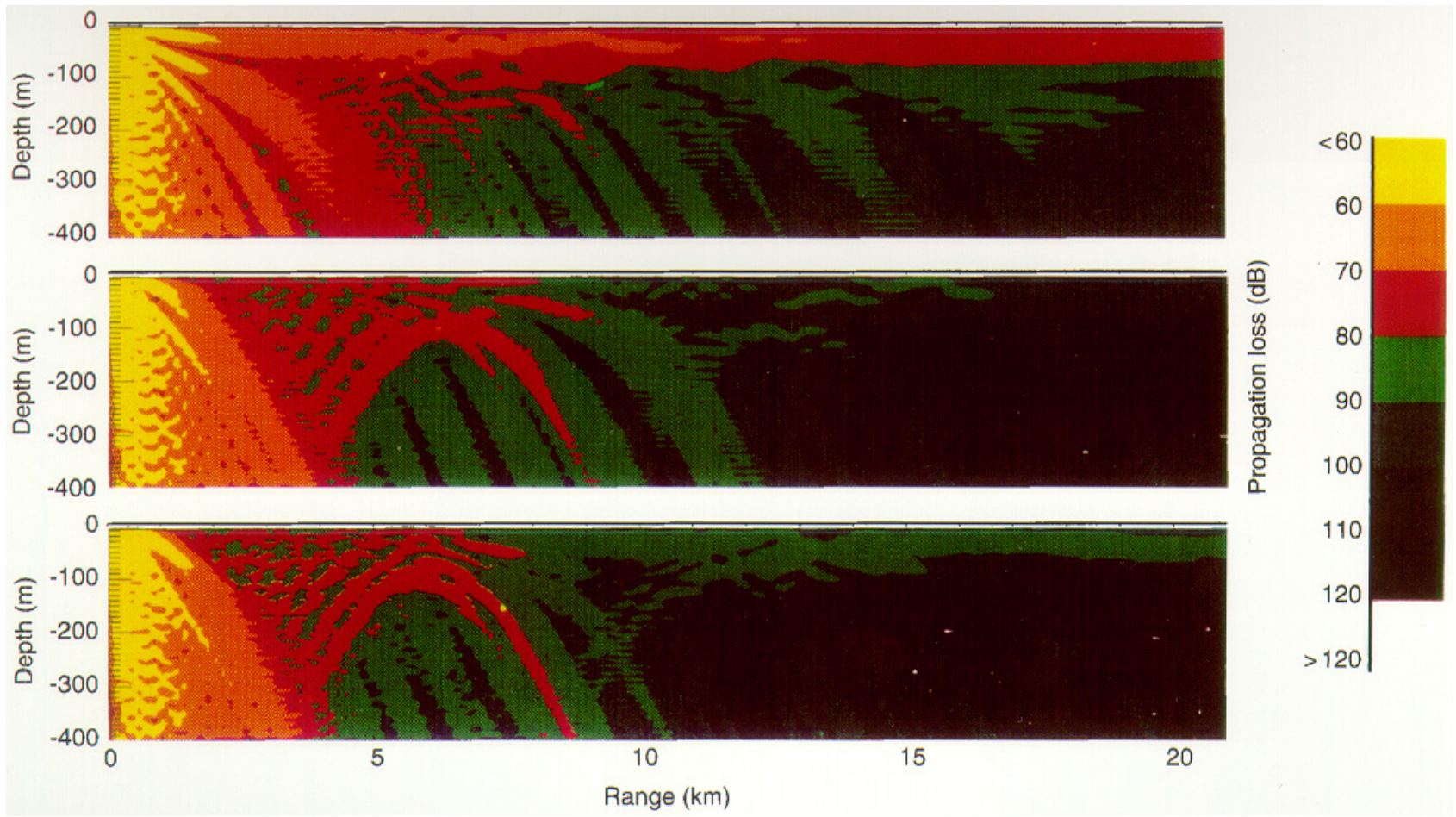

Fig. 10. Propagation-loss contours through the eddy environment, frequency $400 \mathrm{~Hz}$, source depth $50 \mathrm{~m}$. Top panel range-independent background, middle panel feature model, bottom panel observed case

Table 2. Propagation loss error statistics

\begin{tabular}{|c|c|c|c|c|c|}
\hline & \multirow[t]{2}{*}{ Frequency $(\mathrm{Hz})$} & \multicolumn{2}{|c|}{ Unsmoothed results } & \multicolumn{2}{|c|}{ Smoothed results } \\
\hline & & rms error $(\mathrm{dB})$ & $\begin{array}{l}\% \text { over } 10-\mathrm{dB} \\
\text { difference }\end{array}$ & rms error $(\mathrm{dB})$ & $\begin{array}{l}\% \text { over } 10-\mathrm{dB} \\
\text { difference }\end{array}$ \\
\hline \multicolumn{6}{|c|}{ a for eddy environment } \\
\hline Range independent & $\begin{array}{r}50 \\
400\end{array}$ & $\begin{array}{r}7.6 \\
10.5\end{array}$ & $\begin{array}{l}18 \\
43\end{array}$ & $\begin{array}{l}6.4 \\
9.1\end{array}$ & $\begin{array}{l}12 \\
34\end{array}$ \\
\hline Feature model & $\begin{array}{r}50 \\
400\end{array}$ & $\begin{array}{l}5.5 \\
7.1\end{array}$ & $\begin{array}{r}8 \\
15\end{array}$ & $\begin{array}{l}4.4 \\
4.5\end{array}$ & $\begin{array}{l}4 \\
4\end{array}$ \\
\hline \multicolumn{6}{|c|}{$b$ for frontal environment } \\
\hline Range independent & $\begin{array}{r}50 \\
400\end{array}$ & $\begin{array}{l}15.5 \\
15.6\end{array}$ & $\begin{array}{l}52 \\
45\end{array}$ & $\begin{array}{l}15.1 \\
14.4\end{array}$ & $\begin{array}{l}52 \\
38\end{array}$ \\
\hline Feature model & $\begin{array}{r}50 \\
400\end{array}$ & $\begin{array}{l}6.8 \\
9.7\end{array}$ & $\begin{array}{l}12 \\
26\end{array}$ & $\begin{array}{l}5.5 \\
6.7\end{array}$ & $\begin{array}{r}8 \\
10\end{array}$ \\
\hline
\end{tabular}

\subsubsection{Frontal environment}

The propagation-loss curves for a source and receiver at $50-\mathrm{m}$ depth are shown in Fig. $11 \mathrm{a}, \mathrm{b}$. Both the $50-\mathrm{Hz}$ (Fig. 11a) and 400-Hz (Fig. 11b) cases indicate that the range-independent case predicts significantly lower propagation loss beyond the front than the other two cases. This is also seen for a receiver at $150 \mathrm{~m}$ (Fig. 11c). Once again, however, errors due to the internal-wave field are notable when comparing the feature-model and observed curves, due to nulls of intensity being out of phase. At $400 \mathrm{~Hz}$ (Fig. 11b, c), a ducting pattern can be seen in the range-independent case. This is shown more clearly in Fig. 12, which illustrates the $2 \mathrm{D}$ field of propagation-loss values through the three fields at $400 \mathrm{~Hz}$, for a source at $50 \mathrm{~m}$. The range-independent case predicts ducting through the warm water, due to the presence of a weak sound channel at the depth of the source $(50 \mathrm{~m}$ : see Fig. $8 b)$. In contrast, the presence of the frontal feature in the observed and feature-model cases disrupts the surface ducting pattern at around $10 \mathrm{~km}$, refracting sound downwards as in the eddy case, as once again the sound passes through highly stratified cold water.

Rms calculations for the smoothed and unsmoothed propagation-loss fields for the frontal environment are shown in Table $2 \mathrm{~b}$. Following the same reasoning used for Table $2 \mathrm{a}$, it can be seen that much of the feature-model 

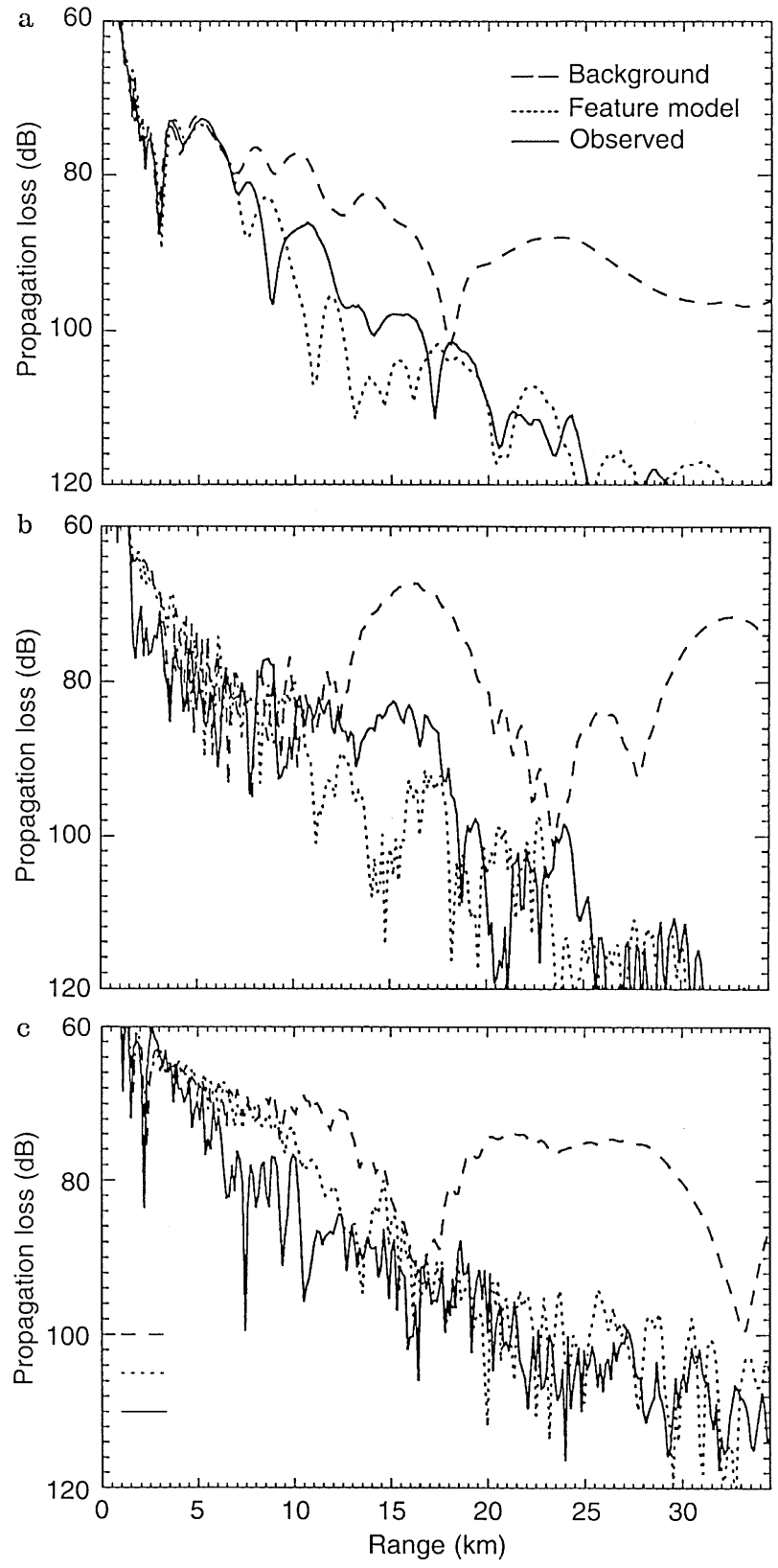

Fig. 11a-c. Propagation-loss values obtained through the frontal environment: a frequency $50 \mathrm{~Hz}$, source and receiver depth $50 \mathrm{~m}$; b frequency $400 \mathrm{~Hz}$, source and receiver depth $50 \mathrm{~m}$; c frequency $400 \mathrm{~Hz}$, source depth $50 \mathrm{~m}$ and receiver depth $150 \mathrm{~m}$

inaccuracies at the higher frequency are once again due to random spikes in the propagation-loss fields, and that overall the feature model reproduces the main (smoothed) acoustic features to within $6 \mathrm{~dB}$ at $50 \mathrm{~Hz}$, and $7 \mathrm{~dB}$ at $400 \mathrm{~Hz}$, compared to $16 \mathrm{~dB}$ for the range-independent case.

\section{Summary and conclusions}

The significant acoustic effects of strong eddies and fronts on changing propagation and ray patterns have been studied before, both for observed and modelled features. Excellent reviews of these studies are given in Kamenkovich et al. (1986) and Robinson and Lee (1994). This paper has confirmed these findings using high-resolution data from DRA hydrographic surveys. The main findings of this paper, however, are related to the effectiveness of feature models, and the impact of observed internal variability.

The acoustic skill of feature modelling was tested by using the technique on high-resolution temperature and lower-resolution salinity data from the Iceland-Faeroes region, which resolved internal waves with wavelengths of order $100 \mathrm{~m}$. Acoustic propagation-loss patterns were compared between the observed data, a feature model of that data and a range-independent environment, for a source depth of $50 \mathrm{~m}$ and frequencies of 50 and $400 \mathrm{~Hz}$. The results showed that the feature models were significant improvements on the range-independent environment in simulating the propagation loss through the observed data. In some cases the range-independent environment gave rms difference errors in propagation loss (relative to the observed case) consistently around $15 \mathrm{~dB}$, and totally misrepresented the pattern of propagation. The feature models captured the overall 'smoothed' features of the observed acoustic patterns, with generally smaller rms errors ( $7 \mathrm{~dB}$ or less); however, some occasional peak (noisy) errors over $10 \mathrm{~dB}$ occurred, in one case over $26 \%$ of the whole field.

These errors in the feature-model predictions are most likely due to the effect of internal waves and fine structure, not represented in the feature model, and confirm previous findings from modelled environments (Small, 1994; Chin-Bin et al., 1994) that internal waves can have at least $5-10-\mathrm{dB}$ effects on propagation loss, additional to the mesoscale effects. The internal waves are difficult to predict deterministically, as they occur on scales and frequencies which are too small to be observed sufficiently for initialising models.

A more likely means of modelling internal waves is by using a stochastic method. The authors (Small, 1994) have recently been investigating this by creating an ensemble of possible internal-wave fields using random frequencies and amplitudes derived from the theory of Garrett and Munk $(1972,1975)$. Propagation-loss values were calculated through each of these fields, and then means and standard deviations of quantities such as convergence zone strength and range were calculated over the ensemble. This method could be used to assign confidence limits to propagation-loss values derived from feature models

In conclusion, feature models have been found to represent correctly the gross features of sound propagation patterns that occur in the real ocean, and improve significantly on situations in which conditions are assumed uniform with range, i.e. where mesoscale effects are ignored. For a complete representation of acoustic variability, however, this study has shown that it might be necessary to combine feature models with stochastic internal wave models to describe the mean acoustic fields and assign confidence limits to the propagation loss estimates. 


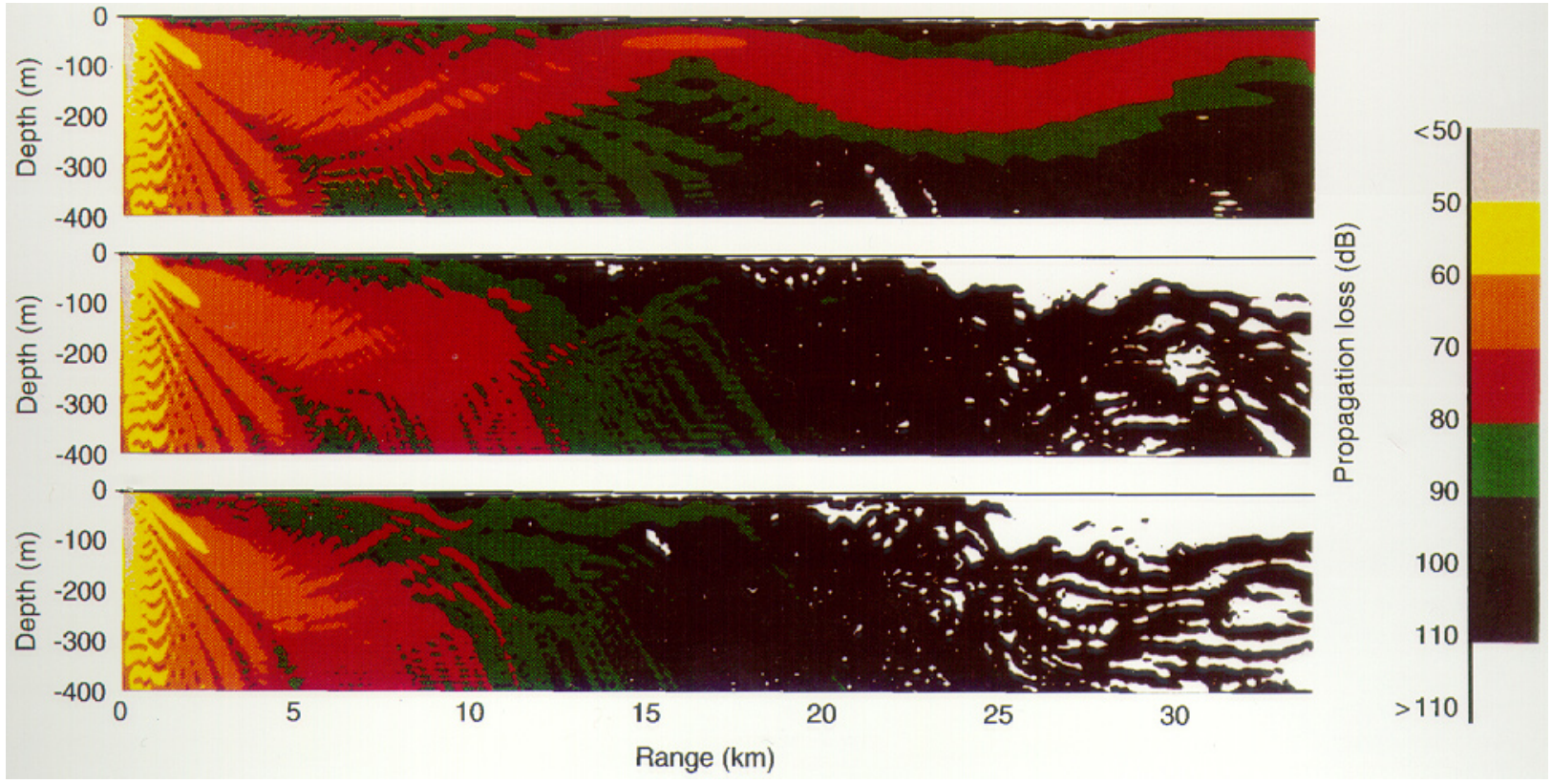

Fig. 12. Propagation-loss contours through the frontal environment, frequency $400 \mathrm{~Hz}$, source depth $50 \mathrm{~m}$. Top panel range-independent background, middle panel feature model, bottom panel observed case

Acknowledgements. Our thanks go to Kate Kelly for providing the DRA data and Sam Marks for advice on the geoacoustic parameters. Topical Editor D. Webb thanks M. Hall and another referee for their help in evaluating this paper.

\section{References}

Chen, C. T., and F. J. Millero, Speed of sound in seawater at high pressures, J. Acoust. Soc. Am., 62 (5), 1129-1135, 1977.

Chin-Bing, S. A., D. B. King, and J. D. Boyd, The effects of ocean environmental variability on underwater acoustic propagation forecasting, in Oceanography and Acoustics, Eds. A. R. Robinson and D. Lee, American Institute of Physics, New York, pp. 7-49, 1994.

Garret, C., and W. H. Munk, Space-time scales of internal waves, Geophys. Fluid Dyn., 3, 225-264, 1972

Garrett, C., and W. H. Munk, Space-time scales of internal waves: A progress report, J. Geophys. Res., 80, 291-297, 1975.

Geddes, N. R., and J. C. Scott, Fivers 91 The RV Brucella thermistor chain dataset: an introduction, DRA internal document TM (USSF) 93125, 1993.

Harrison, C. H., and J. A. Harrison, A simple relationship between frequency and range averaging for broadband sonar, J. Acoust Soc. Am., 97(2), 1314-1318, 1995.

Heathershaw, A. D., and S. J. Foreman, Developments in Oceanographic computer forecasting for the Royal Navy, Ocean Challenge, 5, 45-56, 1994.

Hopkins, T. S., The GIN sea - A synthesis of its physical oceanography and literature 1972-1985, Earth Sci. Rev., 30, 175-318, 1991.

Jensen, F. B., and M. G. Martinelli, The SACLANTCEN parabolic equation model (PAREQ), The SACLANT Undersea Research Centre, La Spezia, Italy, 1985

Jensen, F. B., W. A. Kuperman, M. B. Porter, and H. Schmidt, Computational ocean acoustics, Chapter 6, American Institute of Physics, New York, 1994.

Kamenkovich, V. M., M. N. Koshlyakov, and A. S. Monin, Synoptic eddies in the ocean, D. Riedel, Dordrecht, 1986.
Lane, N. M., and J. C. Scott, The ARE digital thermistor chain, in Proc. Mar. Data Syst. Int. Symp., pp. 127-132, Gulf Section, Marine Technology Society, Bay Saint Louis Miss., 1986.

Maskell, S. J., A. D. Heathershaw, and C. E. Stretch, Topographic and eddy effects in a primitive equation model of the IcelandFaeroes Front, J. Mar. Sys., 3, 343-380, 1992.

Numerical Algorithms Group (NAG), NAG Fortran Library Introductory Guide Mark 16, NAG, Wilkinson House, Jordan Hill Road, Oxford, 1993.

Pedlosky, J., Geophysical fluid dynamics (2nd ed.) Springer-Verlag, Berlin, Heidelberg, New York, 1987.

Robinson, A. R., and D. Lee (eds), Oceanography and Acoustics, American Institute of Physics, New York, 1994.

Scott, J. C., Iceland-Faeroes 1988 - an introduction to the thermistor chain dataset, Internal document, DRA (Mar) TM (USSF) 91151, 1991.

Scott, J. C., and N. M. Lane, Frontal boundaries and eddies on the Iceland Faeroes ridge, in Ocean Variability and Acoustic Propagation, Eds. Potter and Warn-Varnas, Kluwer, London, 449-461, 1991.

J. C. Scott., and A. L. McDowell, Cross frontal cold jets near Iceland in-water, AVHRR, and GEOSAT altimeter data, J. Geophys. Res., 95, C10, 18005-18014, 1990.

Small, J., The effect of random internal wave fields on acoustic propagation, Paper given at UK Oceanography, Stirling University, 1994.

Smeed, D. A., Feature models and data assimilation, Proc. World Meteorol. Organ. (WMO) Int. Symp. Assimilation of Observations in Oceanography and Meteorology, Tokyo, March 1995.

Smeed, D. A., and S. G. Alderson, Inference of deep ocean structure from upper ocean measurements, accepted by J. Atmos. Oceanic Tech., 1995.

Spall, M. A., and A. R. Robinson, Regional primitive equation studies of the Gulf Stream meander and ring formation region, $J$ Phys. Ocean, 20, 985-1016, 1990.

Urick, R. J., Principles of underwater sound (2nd ed.) McGraw Hill, New York, 1983. 